\title{
Ethnic differences in vitamin D and Interleukin-6 levels in healthy South Asian and Caucasian women revealed by the D2-D3 study
}

\author{
A.L. Salter, J. Elliott, J. Sier, O.A. Akinyemi, J. Hunt, S.A. Lanham-New and D.J. Blackbourn \\ School of Biosciences and Medicine, Faculty of Health and Medical Sciences, University of Surrey, Guildford, Surrey, \\ $U K$, GU2 $7 X H$
}

Vitamin D is most commonly known for its importance to bone health, but growing evidence suggests that it also has a role in regulating immune function and the inflammatory response. Vitamin D supplementation has recently been shown to reduce the risk of acute respiratory tract infection ${ }^{(1)}$ and greater levels of circulating vitamin D are associated with a lower risk of chronic disease such as multiple sclerosis ${ }^{(2)}$. Interleukin-6 (IL-6) is an immune function marker, released in the inflammatory response and has been shown to be inhibited by vitamin $\mathrm{D}^{(3)}$. The present study aimed to determine any ethnic differences in IL-6 and serum 25-hydroxyvitamin D (25OHD) at baseline between South Asian and Caucasian women.

A total of 54 subjects (South Asian $n=22$, Caucasian $n=32$ ) were selected from the D2-D3 study ${ }^{(4)}$ based on the greatest $250 H D$ changes across the 12 week intervention period. Plasma samples were analysed from Visit 1 (baseline). IL-6 was quantified using highsensitivity enzyme-linked immunosorbent assay (R\&D Systems, Minneapolis, MN, USA) and all standards and samples were plated in duplicate. Serum 25OHD levels had previously been determined and were therefore available for use in this study. The D2-D3 study had ethical approval from both the South-East Coast (Surrey) NHS Research Ethics Committee (NHS REC No. 11/LO/ 0708) and the University of Surrey Ethics Committee (Fast-Track EC/2011/97/FHMS).

At baseline, IL-6 concentration was significantly higher in the South Asian women (1.83pg/mL IQR 0.85) compared to the Caucasian women $(1.24 \mathrm{pg} / \mathrm{mL} \mathrm{IQR} \mathrm{0.75)}(\mathrm{P}=0.023)$ and serum $25 \mathrm{OHD}$ was significantly lower in the South Asian women $(28.09$ $\pm 15.8 \mathrm{nmol} / \mathrm{L})$ compared to the Caucasian women $(55.98 \pm 24.8 \mathrm{nmol} / \mathrm{L})(\mathrm{P}<0.001)$. No correlations (negative or positive) were found between serum 25OHD and IL-6 in either the South Asian $(r-0 \cdot 186, p=0 \cdot 407)$ or Caucasian $(r-0 \cdot 090$, $p=0 \cdot 625)$ women.

\begin{tabular}{|c|c|c|c|c|c|c|c|c|c|}
\hline & \multicolumn{4}{|c|}{ South Asian $(n=22)$} & \multicolumn{4}{|c|}{ Caucasian $(n=32)$} & \multirow[b]{2}{*}{$P$ value } \\
\hline & Mean & SD & Median & IQR & Mean & $\mathrm{SD}$ & Median & IQR & \\
\hline $25 \mathrm{OHD}(\mathrm{nmol} / \mathrm{L})$ & 28.09 & $15 \cdot 77$ & $22 \cdot 20$ & $24 \cdot 67$ & $55 \cdot 98$ & $24 \cdot 76$ & $48 \cdot 75$ & $41 \cdot 90$ & $<0.001^{*}$ \\
\hline IL-6 (pg/mL) & 1.99 & 1.03 & $1 \cdot 83$ & $0 \cdot 85$ & 1.48 & $0 \cdot 64$ & $1 \cdot 24$ & $0 \cdot 75$ & $0.023 \dagger$ \\
\hline
\end{tabular}

$* \mathrm{P}<0.05$ Independent Samples T-test, $\dagger \mathrm{P}<0.05$ Mann Whitney U test, IQR, Interquartile Range.

The present study shows a significant ethnic difference in both IL-6 and serum 25OHD concentrations at baseline with South Asian women having greater IL-6 and lower 25OHD levels than Caucasian women. Very few studies have looked at IL-6 and vitamin D in a UK-based South Asian population, although a cross-sectional study carried out in West London found that IL-6 was significantly higher in South Asian women compared to European women ${ }^{(5)}$. An ethnic difference in serum 25OHD levels between South Asian and Caucasian women has previously been identified, with South Asian women being identified as vitamin D deficient ${ }^{(6)}$. The present study supports both of these previous findings. Further work is underway to investigate the effect of vitamin D supplementation, compared to placebo, on Interleukin-6 and serum 25OHD after 6 and 12 weeks intervention in the selected participants.

1. Martineau AR, Jolliffe DA, Hooper RL et al. (2017) BMJ 356, i6583.

2. Munger KL, Levin LI, Hollis BW et al. (2006) JAMA 296, 2832-2838.

3. Zhang Y, Leung DYM, Richers BN et al. (2012) J Immunol 188, 2127-2135.

4. Tripkovic L, Wilson L, Hart K et al. (2015) Proc Nutr Soc 74, OCE1, E16.

5. Peters MJL, Ghouri N, McKeigue P et al. (2013) Cytokine 61, 29-32.

6. Darling AL et al. (2013) Osteoporos Int 24(2): 477-488. 